\title{
Evaluation of the anti-fatigue effects of a traditional herbal drug, Gongjin-dan, under insufficient sleep conditions: study protocol for a randomised controlled trial
}

Mi Ju Son', Hwi-Jin $\mathrm{Im}^{2}$, Young-Eun $\mathrm{Kim}^{3}$, Boncho Ku${ }^{4}$, Jun-Hwan Lee ${ }^{1,5^{*}}$ (D) and Chang-Gue Son ${ }^{2^{*}}$

\begin{abstract}
Background: Many herbal medicines are traditionally used as anti-fatigue agents in east Asian countries; however, there is a dearth of clinical evidence supporting the anti-fatigue effects of such medicines and their mechanisms. This study is a feasibility trial to assess the clinical efficacy of Gongjin-dan (GJD) and verify its mechanisms by exploring fatigue outcomes, including endocrine and immunological biomarkers in humans.

Methods/Design: To investigate the anti-fatigue effects of GJD and the mechanism underlying these effects, a randomised, double-blind, placebo-controlled crossover clinical trial was designed. Participants ( 24 healthy male volunteers) will be hospitalised for 4 days (3 nights), during which acute fatigue and stress conditions will be induced by sleep deprivation, and GJD or a placebo will be administered (twice daily). The primary outcome will be changes in serum cortisol levels, measured in the morning, as an objective biomarker of sleep deprivation-induced fatigue and stress. The secondary outcomes will include: the Fatigue Severity Scale; the Brief Fatigue Inventory, and the Leeds Sleep Evaluation Questionnaire scores; levels of salivary cortisol, epinephrine, norepinephrine, oxidative stressrelated biomarkers, homocysteine, and immunological factors; and heart rate variability. After a washout period of more than 4 weeks, a second treatment phase will commence in which participants who were previously administered the placebo will receive the drug and vice versa, following the same treatment regime as in the first phase.
\end{abstract}

Discussion: This study protocol provides a unique opportunity to enhance our understanding of fatigue and the effects of GJD on fatigue in terms of endocrine and immunological mechanisms by validating the study design and determining feasibility. Findings from this trial will help researchers to design a pilot or definitive clinical trial of traditional herbal medicine for chronic fatigue.

Trial registration: Korean National Clinical Trial Registry CRIS; KCT0001681, registered on 29 October 2015.

Keywords: Fatigue, Traditional herbal medicine, Randomised controlled trial, Protocol

Abbreviations: ALT, Alanine aminotransferase; ANOVA, Analysis of variance; AST, Aspartate aminotransferase; BFI-K, Brief Fatigue Inventory-Korean version; CAM, Complementary and alternative medicine; CV, Coefficient variation; ECG, Electrocardiogram; ELISA, Enzyme-linked immunosorbent assay; FAS, Full analysis set; FSS-K,

\footnotetext{
* Correspondence: omdjun@kiom.re.kr; ckson@dju.ac.kr

${ }^{1}$ Clinical Research Division, Korea Institute of Oriental Medicine, 1672

Yuseongdae-ro, Yuseong-gu, Daejeon 34054, Republic of Korea

${ }^{2}$ Liver and Immunology Research Center, Daejeon Korean Medicine Hospital

of Daejeon University, 176-9, Daeheung-ro, Jung-gu, Daejeon 34929,

Republic of Korea

Full list of author information is available at the end of the article
} 


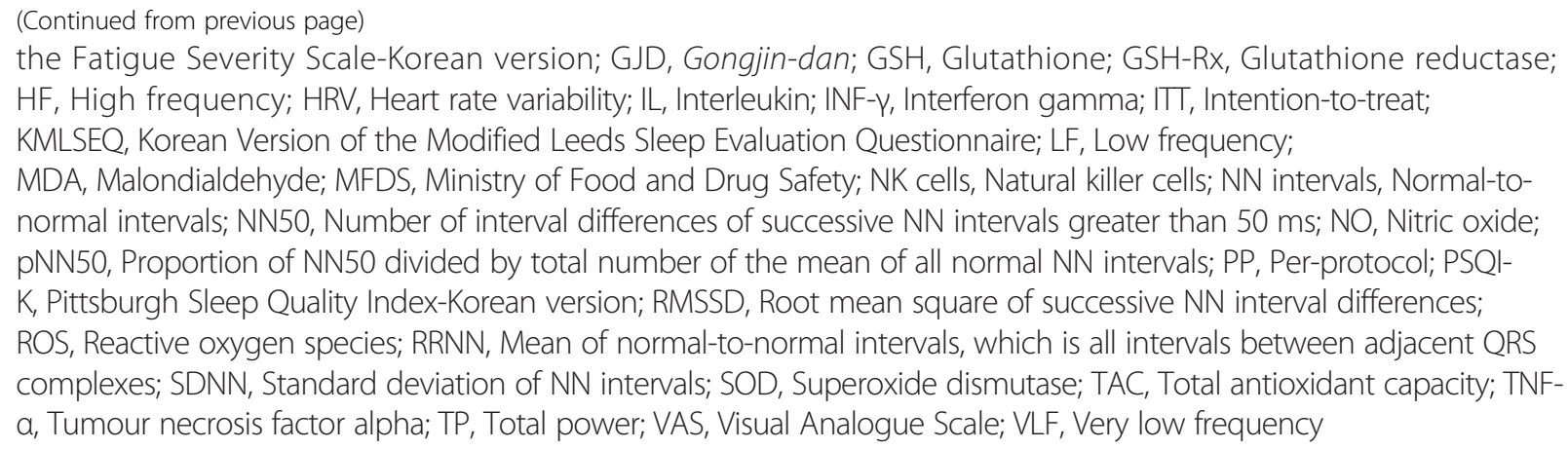

\section{Background}

Fatigue is a subjective symptom of lethargy experienced during or after performing daily work, or a feeling of a lack of energy sufficient to affect daily life. The average worldwide incidence of chronic fatigue is $11.1 \%$ and most fatigue resolves with rest; however, treatment is required when fatigue is prolonged and reduces the quality of life of individuals and their families, or generally affects society $[1,2]$. Among people with chronic fatigue, labor force productivity is known to decrease by $54 \%$ and the cost of lost productivity is estimated to be $\$ 9.1$ billion annually in the US. [3].

Fatigue is a multifactorial condition and the pathophysiological mechanisms of idiopathic chronic fatigue or chronic fatigue syndrome are unclear. For this reason, there is no standard treatment for chronic fatigue [4] and some patients with chronic fatigue, therefore, turn to complementary and alternative medicine (CAM) for treatment $[5,6]$. In East Asian countries, CAM in the form of traditional herbal medicine is widely used to treat fatigue [7, 8]; the traditional herbal drug, Gongjin-dan (GJD), is generally prescribed by Korean traditional medicine physicians to treat patients with chronic fatigue in Korean traditional medicine clinics. Although the antifatigue effects of herbal medicines, including GJD [9], deer antler extract [10], and tao-hong-si-we-tang [11], have been demonstrated in animal studies, few clinical studies have focussed on these treatments. To encourage evidence-based practices in the traditional medicine field, well-designed studies must be conducted to evaluate the effects and mechanisms of action of traditional herbal medicines, including traditional anti-fatigue medicines.

This study is a feasibility trial to assess the clinical efficacy of GJD and verify its mechanisms by exploring fatigue outcomes, including endocrine and immunological biomarkers. The unique protocol described herein is designed to minimise confounding factors and other possible bias, and comprises a randomised, double-blinded, placebo-controlled crossover clinical trial with hospitalised subjects. However, the findings from this trial cannot be generalised to individuals suffering with chronic fatigue syndrome because sleep-deprived healthy men aged 19-45 years were chosen as the participants of this study to avoid confounding factors and improve internal validity.

This study design may serve as a guide for researchers seeking to effectively evaluate the effects and underlying mechanisms of traditional herbal anti-fatigue medicines as well as conventional drugs.

\section{Methods \\ Objective}

The aim of this study was to develop a protocol to (1) evaluate the anti-fatigue effects of GJD in acute fatigue; (2) verify the mechanisms underlying the observed effects by assessing changes in stress hormones, oxidative stress-related biomarkers, homocysteine, and immunological factors; and (3) clinically assess the effectiveness and safety of GJD in treating acute fatigue using the Korean version of the Fatigue Severity Scale (FSS-K), Brief Fatigue Inventory (BFI-K), and Leeds Sleep Evaluation Questionnaire (KMLSEQ) scores as well as carrying out daily fatigue and sleep evaluations and assessments of heart rate variability (HRV) and adverse events.

\section{Design and setting}

This randomised, double-blind (both patient and practitioner or assessor), placebo-controlled crossover clinical trial will be conducted in Republic of Korea.

\section{Recruitment period}

Participant recruitment began in September 2015 at the Daejeon Korean Medicine Hospital of Daejeon University, and it is expected to be completed in December 2015.

\section{Methods of recruitment}

Twenty-four participants will be recruited for this trial, and participants will be recruited using online and printed advertisement of the study. Advertisements will be posted on the online bulletin board of the Daejeon Korean 
Medicine Hospital of Daejeon University, and printed advertisements will be posted in stores in university towns and on school bulletin boards. Advertisements will also be posted on online social networking platforms. The relevant trial information will be conveyed to the potential participants prior to their first visit to the hospital.

\section{Study plan}

Subject information will be collected after oral and written consent is obtained from each participant at the first visit.

Screening phase After potential participants voluntarily consent to the study, they will be screened using predetermined inclusion/exclusion criteria at the first visit. The participants will be instructed to sleep for at least $7 \mathrm{~h}$ daily for 1 week before admission and to avoid new medications, alcohol, and caffeine consumption for 2 days before admission. Participants will be randomly allocated to one of two groups. The group allocation will be concealed from both the participants and the practitioners.

Treatment and evaluation phase Both the treatment and placebo-control groups will be hospitalised for
4 days, and after a washout period of over 4 weeks the participants will be hospitalised again for 4 days. During hospitalisation, subjects will be administered GJD or placebo (one pill twice a day $30 \mathrm{~min}$ before breakfast and dinner) for 3 days. Group A participants will be administered GJD in phase I, followed by placebo treatment in phase II (both treatments will be administered twice daily for 3 days each). Group B participants will be administered placebo in phase I, followed by GJD treatment in phase II (same regimen as in group A; Fig. 1). To assess the effects of GJD treatment, participants will be allowed to sleep for only $4 \mathrm{~h}$ (02:00 to 06:00) on days 2 and 3 to artificially induce fatigue, and will carry out various activities such as walking, taking lectures, and watching movies to limit daytime sleep. To control possible bias, all subjects will be limited to intensive exercise and will consume the same foods during the study periods.

Blood samples will be collected at 06:30 for analysis of serum cortisol, stress hormones, oxidative stress-related biomarkers, homocysteine, and immunological factors. Saliva samples will be collected at 06:30, 12:00, and 21:00 for analysis of salivary cortisol, while HRV will be assessed

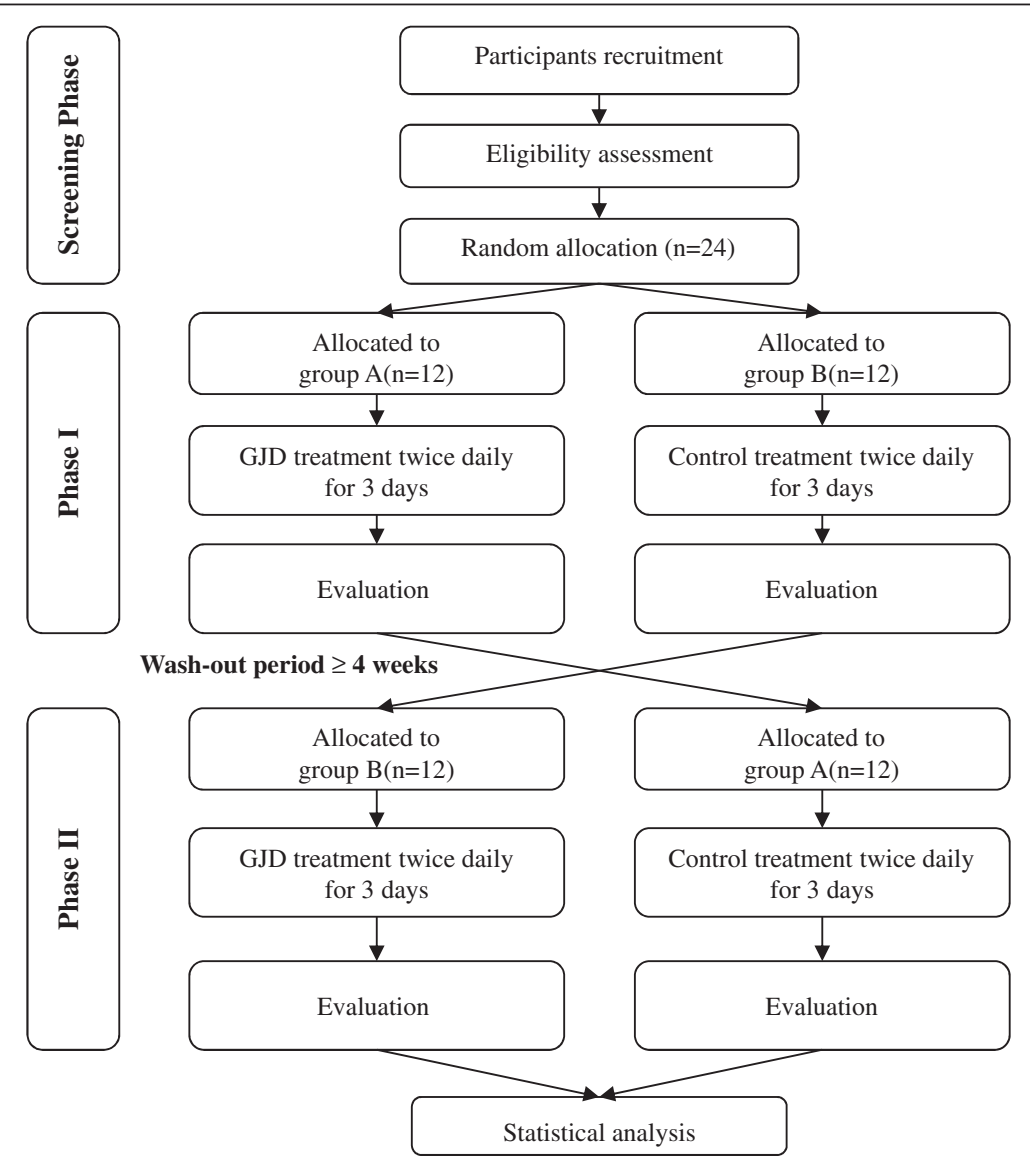

Fig. 1 Flowchart describing the study plan 
at 15:00-17:00. Self-reporting of FSS-K, BFI-K, KMLSEQ, and daily fatigue and sleep status will be carried out via questionnaires after dinner on days $1,2,3$, and 4 .

After a 4-week washout period, participants will be admitted to phase II for 4 days, during which they will undergo the same schedule.

The schedule of events for the trial is outlined in Table 1 and the study participants' timeline of activities, treatments, and assessments during hospitalisation is shown in Fig. 2.

\section{Study participants \\ Inclusion criteria}

Participants are eligible for inclusion in the study if they meet the following criteria:
1. Healthy male volunteers aged 19 to 45 years at the screening visit

2. Participants must weigh over $45 \mathrm{~kg}$, with a body mass index of $18.5-30 \mathrm{~kg} / \mathrm{m}^{2}$ at the screening visit

3. Participants must not have any congenital defects or chronic diseases that have been present within the 3 years prior to the screening visit and should have no pathological or abnormal clinical findings on physical examination

4. Participants must not have any sleep disorders and must have Pittsburgh Sleep Quality Index (Korean version; PSQI-K) scores of 5 points or lower

5. Participant must keep normal nocturnal sleeping hours (between 21:00 and 09:00) for a week before

Table 1 Schedule of treatments and outcome measurements

\begin{tabular}{|c|c|c|c|c|c|c|c|c|c|}
\hline Step of trial & Screening & \multicolumn{8}{|c|}{ Treatment and evaluation } \\
\hline Phase & & \multicolumn{4}{|c|}{ Phase I (1st Admission) } & \multicolumn{4}{|c|}{ Phase II (2nd Admission) } \\
\hline Day & & 1 & 2 & 3 & 4 & 1 & 2 & 3 & 4 \\
\hline Informed Consent & 0 & & & & & & & & \\
\hline Demographic Characteristics & ○ & & & & & & & & \\
\hline Physical Examination & ○ & & & & & & & & \\
\hline Medical history & - & & & & & & & & \\
\hline Inclusion/Exclusion criteria & • & & & & & & & & \\
\hline Complete Blood Count (CBC) & 0 & & & & 0 & & 0 & & $\bigcirc$ \\
\hline Liver Function Test (LFT) & - & & & & 0 & & & & $\bigcirc$ \\
\hline Urine Analysis (UA) & ○ & & & & 0 & & & & $\bigcirc$ \\
\hline Chest Radiograph & ○ & & & & & & & & \\
\hline Electrocardiogram & $\bullet$ & & & & & & & & \\
\hline Vital signs & 0 & $\mathbf{\square}$ & $\mathbf{\square}$ & $\mathbf{\square}$ & $\mathbf{\square}$ & $\mathbf{\square}$ & - & - & $\mathbf{\square}$ \\
\hline PSQI-K & $\bullet$ & & & & & & & & \\
\hline FSS-K & ○ & $\mathbf{\square}$ & & & $\mathbf{\square}$ & $\mathbf{\square}$ & & & $\mathbf{\square}$ \\
\hline Randomisation & & ○ & & & & & & & \\
\hline $\begin{array}{l}\text { Stress hormones, oxidative } \\
\text { stress-related biomarkers }\end{array}$ & & & O & 0 & O & & O & $\bigcirc$ & $\bigcirc$ \\
\hline Immunologic factors $^{\mathrm{b}}$ & & & 0 & & 0 & & 0 & & O \\
\hline Homocysteine & & & 0 & & 0 & & 0 & & $\bigcirc$ \\
\hline Salivary cortisol & & () & (a) & (a) & () & (2) & (2) & (a) & () \\
\hline BFI-K & & $\mathbf{\square}$ & $\boldsymbol{\square}$ & $\mathbf{\square}$ & $\mathbf{\square}$ & $\mathbf{\square}$ & a & $\mathbf{\square}$ & $\mathbf{\square}$ \\
\hline KMLSEQ & & $\mathbf{\square}$ & $\mathbf{\square}$ & $\mathbf{\square}$ & $\mathbf{\square}$ & $\mathbf{\square}$ & $\mathbf{\square}$ & $\mathbf{\square}$ & $\mathbf{\square}$ \\
\hline $\begin{array}{c}\text { Daily Sleep and Fatigue Status } \\
\text { Questionnaire }\end{array}$ & & & $\mathbf{\square}$ & $\mathbf{\square}$ & & & 口 & ש & $\mathbf{\square}$ \\
\hline Heart rate variability & & $\square$ & $\square$ & $\square$ & $\square$ & $\square$ & $\square$ & $\square$ & $\square$ \\
\hline Meal & & $\square$ & $\square$ & $\square$ & $\square$ & 回 & $\square$ & $\square$ & $\square$ \\
\hline Medication & & & $\diamond$ & $\diamond$ & $\diamond$ & & $\diamond$ & $\diamond$ & $\diamond$ \\
\hline Adverse event assessment & & $\mathbf{\square}$ & $\mathbf{\square}$ & 口 & $\mathbf{\square}$ & $\square$ & 口 & 口 & $\mathbf{\square}$ \\
\hline Drug Compliance assessment & & & ○ & $\bullet$ & $\bullet$ & & ○ & - & - \\
\hline Blinding assessment & & & & & & & & & $\boldsymbol{\square}$ \\
\hline
\end{tabular}

${ }^{a}$ Stress hormones; oxidative stress-related biomarkers: serum cortisol, epinephrine, norepinephrine; ROS, NO, MDA, protein carbonyl, GSH, GSH-Rx, SOD, satalase, TAC

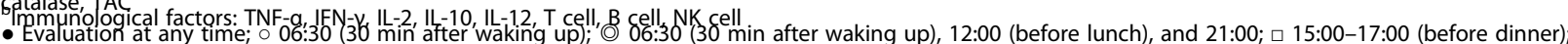
口 07:30-08:30, 12:10-13:00, and 17:30-18:30; 18:30-21:00 (after dinner); $\diamond 1$ pill twice a day, 30 min before breakfast/dinner 


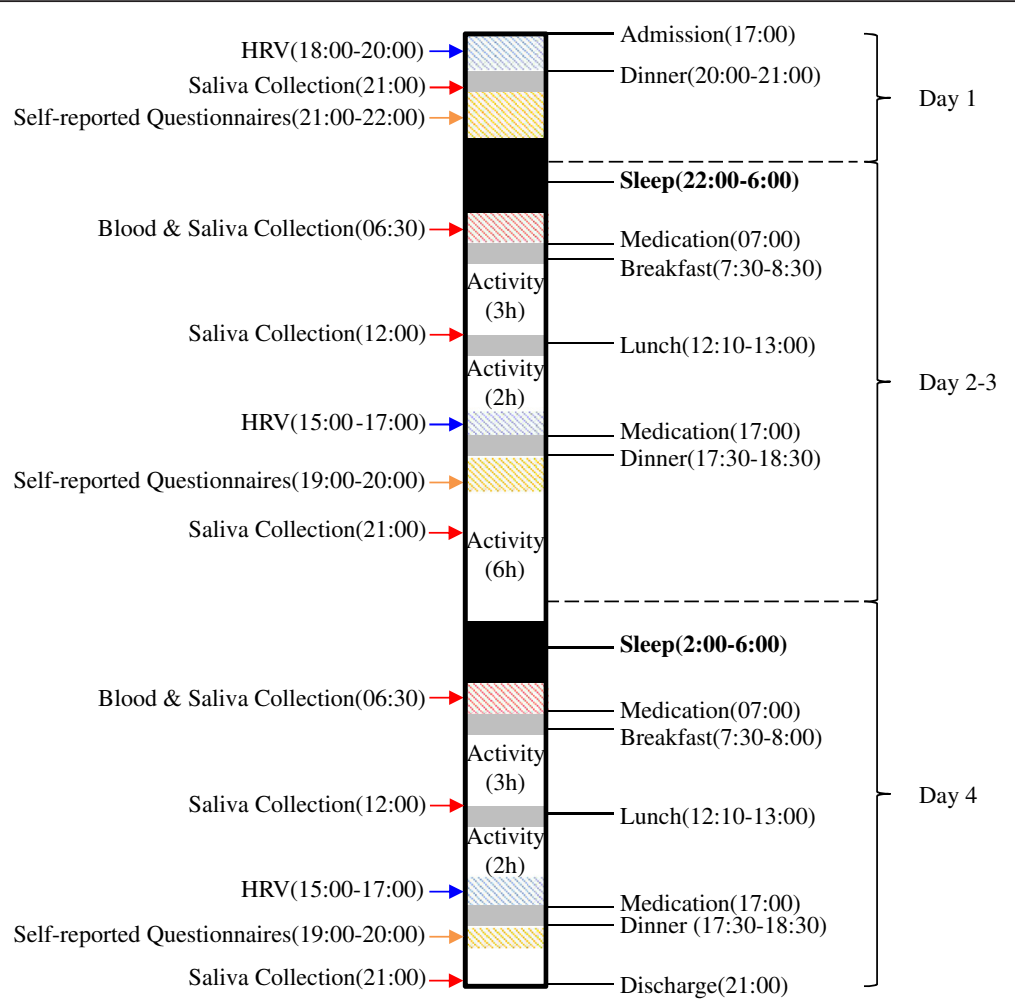

Fig. 2 Timeline of participant activities, treatments, and assessments during each study phase

the first administration of the investigational drug (or placebo)

6. Participants must sleep at least $7 \mathrm{~h}$ per night for a week before the first administration of the investigational drug (or placebo)

7. Participants must be nonsmokers or ex-smokers who stopped smoking at least 1 year before the screening visit

8. Participants must comprehend the purpose and process of the study as well as the properties of the investigational drug and must voluntarily sign a written informed consent approved by the Institutional Review Board of Daejeon Korean Medicine Hospital of Daejeon University

\section{Exclusion criteria}

Participants who experience, have, or have had one or more of the following will be excluded:

1. A clinically significant medical history or a current disease in one of the following areas: cardiovascular, respiratory, gastrointestinal, hepatic, metabolic and endocrine, renal and urinary, reproductive, musculoskeletal, skin and connective tissue, neurological, psychiatric, or allergic (other than seasonal allergies that are asymptomatic at the screening visit) disease
2. A medical history of gastrointestinal diseases (oesophageal achalasia, oesophageal stricture, or Crohn's disease) or operations (other than simple appendectomy or hernia surgeries) that may affect drug absorption

3. Laboratory test results showing alanine aminotransferase (ALT) or aspartate aminotransferase (AST) levels of more than twice the upper limit of the normal range

4. A history of alcohol or drug abuse within the 12 months before the screening visit

5. History of regular alcohol consumption (>210 g/week) within the 6 months before the screening visit. (Beer $(5 \% \mathrm{v} / \mathrm{v}): 250 \mathrm{~mL}=10 \mathrm{~g}$, soju $(20 \% \mathrm{v} / \mathrm{v}): 50 \mathrm{~mL}=8 \mathrm{~g}$, wine $(12 \% \mathrm{v} / \mathrm{v}): 125 \mathrm{~mL}=12 \mathrm{~g})$

6. Participation in another clinical study during the 2 months before the screening visit

7. Treatment with drugs with known effects on drug-metabolising enzymes (e.g. dexamethasone, phenytoin, carbamazepine, rifabutin, rifampicin, phenobarbital, ketoconazole, itraconazole, clarithromycin, atazanavir, indinavir, nelfinavir, ritonavir, clarithromycin, telithromycin, voriconazole, etc.) during the 30 days before the screening visit

8. Whole blood donation during the 2 months before the screening visit, or specific blood component donation in the month before the screening visit 
9. Seated systolic blood pressure $\geq 140 \mathrm{mmHg}$ or diastolic blood pressure $\geq 90 \mathrm{mmHg}$ at the screening visit

10. Intake of prescribed or over-the-counter drugs during the 7 days before the first administration of the investigational drug (or placebo)

11. Consumption of alcohol during the 7 days before the first administration of the investigational drug (or placebo)

12. Serious, acute, or chronic medical or mental conditions or abnormal laboratory findings that may be exacerbated by administration of the investigational drug or participation in the study, or that may affect the study outcomes

13. Hypersensitivity to the investigational drug or any of its components

14. Absence of intention or ability to adhere to the behavioural rules and requirements as specified in the present protocol

15. Any person considered unsuitable for participation in the study by the principal investigator

\section{Intervention}

Trial medications will be prepared by Kyoung-Bang Pharmaceutical Co. Ltd. (Incheon, Republic of Korea) certified in Good Manufacturing Practices of herbal medicine extract pills and granules from the Ministry of Food and Drug Safety (MFDS). Quality control and quality assurance on the quality and safety testing, the packaging, and the contents of the treatment interventions including checks for potential contaminants, such as heavy metals or steroids, will be undertaken by the manufacturers to ensure treatment drug stability and quality.

Quality control and quality assurance regarding the identification of products as well as quality and safety testing will be conducted by the manufacturer. The raw herbs will be washed, dried, ground, and mixed with a pure form of honey $(\mathrm{Mel})$ before being wrapped in gold foil and formed into pills. The pills will be packaged in identically shaped containers weighing $3.75 \mathrm{~g}$ each. The GJD and placebo pills will be formulated to a similar size, shape, taste, and flavour and will be wrapped in gold foil.

GJD consists of three medicinal herbs (Ginseng radix, Korean angelica, Corni fructus), two animal-derived materials (Cornus cervi parvum and Moschus) and Mel. All constituents of the formulation will comply with Korean Pharmacopoeia standards [40]. A placebo will be manufactured by the same pharmaceutical company and will be comprised of corn starch as the main ingredient, as well as small amounts of Dioscoreae rhizoma, Poria sclerotium, $\mathrm{Mel}$, a colouring agent, and a flavouring agent. Each pill will weigh $3.75 \mathrm{~g}$ and the amounts and sources of each component of the formulation are shown in Table 2. The participants will take GJD or placebo twice a day $30 \mathrm{~min}$ before breakfast and dinner, a total of six times during hospitalisation.

During the study period, subjects will not be allowed to receive other fatigue-related treatments such as dietary supplements or alternatives. A practitioner will check to ensure that participants have taken the GJD or placebo immediately after administration and will collect empty bottles in order to confirm that all doses have been taken.

\section{Outcomes}

Detailed outcome measurement time points are provided in Table 1.

\section{Primary outcome}

The primary outcome of this study is the participants' serum cortisol levels. The effect of GJD on serum cortisol will be assessed by observing differences in cortisol levels before (day 2) and after (day 4) administration with GJD or placebo dosing after fatigue has been induced by sleep deprivation in healthy subjects. Considering circadian rhythms, cortisol will be measured at a specific time daily. Serum cortisol levels will be measured on an empty stomach $30 \mathrm{~min}$ after waking, and subjects will not eat foods that might affect cortisol levels, such as those high in sugar, fat, or protein for $12 \mathrm{~h}$ before the first administration of the investigational drug or placebo $[12,13]$.

Table 2 Composition of Gongjin-dan

\begin{tabular}{|c|c|c|c|c|}
\hline Herbal name & & Scientific name (local name) & Quantity (g/pill) & Place of origin \\
\hline \multirow[t]{3}{*}{ Herbs } & Corni fructus & Cornus officinalis Siebold et Zucc. (山荣英) & 0.9 & Korea \\
\hline & Korean angelica & Angelica gigas Nakai (當歸) & 0.9 & Korea \\
\hline & Ginseng radix & Panax ginseng C.A. Meyer (人蔘) & 0.9 & Korea \\
\hline \multirow[t]{2}{*}{ Animal derives } & Moschus & Moschus moschiferus L. (貨香) & 0.15 & Russia \\
\hline & Cornus cervi parvum & Cervus nippon Temminck (鹿茸) & 0.9 & Russia \\
\hline \multirow[t]{2}{*}{ Diluting agents } & Mel & Apis indica Radoszkowski (蜂蜜) & & Korea \\
\hline & Aurum & Gold (金) & & Korea \\
\hline
\end{tabular}




\section{Secondary outcome}

Salivary cortisol levels, the secondary outcome of this study, will be measured once at admission on day 1 at 21:00, and three times daily on days $2-4$; for example, cortisol will be measured within 30 min of waking, at 12:00, and at 21:00. Subjects will not eat within approximately $1 \mathrm{~h}$ or brush their teeth within $10 \mathrm{~min}$ before saliva collection in order to avoid contamination.

Blood samples for analysis of epinephrine, norepinephrine, oxidative stress-related biomarkers (reactive oxygen species (ROS), nitric oxide (NO), malondialdehyde (MDA), protein carbonyl, glutathione (GSH), GSH reductase (GSH-Rx), superoxide dismutase (SOD), catalase, and total antioxidant capacity (TAC)), homocysteine, and immunological factors (tumour necrosis factor (TNF)- $\alpha$, interferon (IFN)- $\gamma$, interleukin (IL)-2, IL-10, IL-12, T cells, B cells, and natural killer (NK) cells) will be collected at the same time as the serum cortisol samples. Complete blood counts will be conducted to quantify the populations of T cells, B cells, and NK cells during phase II, day 2 .

A self-reported questionnaire validated for each purpose and modified to a Korean version will be utilised to measure perceived fatigue. The FSS will be used to measure overall fatigue status [14], the BFI for variation in daily fatigue $[15,16]$, and the LSEQ for variation in sleep aspects [17]. The nine-item FSS is a self-reporting questionnaire developed by Krupp et al. [14] and serves to evaluate fatigue during the past week using statements that the participants must scale from 1 to 7 in terms of degree of agreement. The BFI is a self-reporting questionnaire developed by Mendoza et al. [15] to evaluate fatigue in patients with cancer. This nine-item questionnaire assesses the degree of fatigue at the present moment and for the past $24 \mathrm{~h}$, as well as the impact of fatigue on daily life, on a scale of 0 to 10 . The LSEQ consists of 10 items, each of which is measured on the Visual Analogue Scale (VAS); each item contains opposite statements at both extremes. The LSEQ involves four domains related to sleep: ease of initiating sleep (questions 1, 2, and 3), quality of sleep (questions 4 and 5), ease of waking (questions 6 and 7), and behaviour after waking (questions 8,9 , and 10). To complement the results of the BFI-K and the KMLSEQ, a daily sleep and fatigue status questionnaire developed by our research team will also be completed by the participants. This checks daily sleep and fatigue intuitively on the VAS and is structured so that participants can spontaneously describe a variety of their own symptoms.

In the afternoon of days $1-4$, HRV testing will be performed to study variations in the autonomic nervous system responses relevant to fatigue, where HRV is measured as variations in the beat-to-beat intervals; $\mathrm{R}$ is a point corresponding to the peak of the QRS complex of the electrocardiogram (ECG) wave and RR is the interval between successive Rs. For HRV assessments, $5 \mathrm{~min}$ of ECG signalling will be measured and calculated using a Neo Dinamika system (MR Co. Ltd., Gyeonggi-do, Republic of Korea). The HRV measurements will be taken after participants rest for $10 \mathrm{~min}$, and the subjects will be seated comfortably in a chair in a quiet and bright room at 20 $25{ }^{\circ} \mathrm{C}$ for the assessment. The equipment will be connected to the medial side of both wrists and ankles as well as to the insides of both wrists and the right ankle. Rhythm parameters of the HRV include pulse rate, vegetative balance index, rhythm vegetative factor, regulation process adequacy index, and tension index. Vegetative regulation parameters of the HRV include regulation level and regulation reserves. Statistical analysis of the HRV includes the RRNN (mean of normal-to-normal $(\mathrm{NN})$ intervals, which is all intervals between adjacent QRS complexes); the SDNN (standard deviation of NN intervals); CV (coefficient variation); the RMSSD (the root mean square of successive $\mathrm{NN}$ interval differences); the NN50 (the number of interval differences of successive $\mathrm{NN}$ intervals greater than $50 \mathrm{~ms}$ ); and the pNN50 (the proportion of NN50 divided by total number of the mean of all normal NN intervals). Spectral analysis includes HF (high frequency, 0.15-0.4 Hz), LF (low frequency, $0.04-0.15 \mathrm{~Hz}$ ), VLF (very low frequency, 0.0033 to $0.04 \mathrm{~Hz}$ ), normalised $\mathrm{HF}$, normalised LF, LF/HF ratio and TP (total power).

\section{Laboratory analysis \\ Determining stress hormones}

Serum and salivary levels of cortisol as well as serum levels of norepinephrine and epinephrine will be assessed using enzyme-linked immunosorbent assay (ELISA) kits (LDN GmbH \& Co., Nordhorn, Germany) according to the manufacturer's protocol.

\section{Determining oxidant biomarker levels in serum}

Total serum levels of ROS will be determined according to the method described by Hayashi and colleagues [18]. Serum NO levels will be determined using the Griess method [19] and serum lipid peroxide levels will be determined using thiobarbituric acid (TBA) reactive substances as previously described [20]. The serum levels of protein carbonyl will be measured using a commercial ELISA kit (Cell Biolabs Inc., San Diego, CA, USA) and serum homocysteine levels will be analysed using an auto-analyser (AU400; Olympus, Tokyo, Japan).

\section{Determining antioxidant biomarker levels in serum}

Total antioxidant capacity (TAC) will be determined using a previously described method [21]. The serum levels of total glutathione (GSH) and GSH reductase (GSH-Rx) will be determined using commercial kits (Abcam, Cambridge, MA, USA) and SOD activity in the 
serum will be determined using an SOD assay kit (Dojindo Laboratories, Kumamoto, Japan) according to the manufacturer's protocol. Serum catalase activities will be assayed as previously described [22]

\section{Determining immune-related cytokine levels in serum}

Serum levels of immune-related cytokines will be measured using commercial ELISA kits for TNF- $\alpha$, IFN- $\gamma$ as well as IL-2, IL-10, and IL-12 (BD Biosciences, San Jose, CA, USA). Absorbance will be measured with a spectrophotometer (Molecular Devices, Sunnyvale, CA, USA).

\section{Flow cytometry and haematological analysis}

Lymphocyte subclasses in the peripheral blood will be analysed using a BD Multitest IMK kit with a FACSCalibur instrument and Cell Quest Pro software (BD Biosciences, San Jose, CA, USA). Complete blood counts will be obtained using a Hemavet analyser (CDC Technologies, Dayton, OH, USA). T cells (CD3+), B cells (CD19+), and NK cells (CD3-/CD16+/CD56+) will be quantified as a percentage of the total number of lymphocytes.

\section{Randomisation and allocation concealment}

Randomisation codes will be generated through block randomisation in a 1:1 code for groups A (phase I: GJD, phase II: placebo) and B (phase I: placebo, phase II: GJD). For the generation of a randomisation code, Random Allocation Software program v2.0.0 (M. Saghaei, MD, Department of Anaesthesia, Isfahan University of Medical Sciences, Isfahan, Iran) will be used.

\section{Blinding}

A researcher who is not involved in recruitment or assessment will assign the clinical trial subjects identification numbers based on the randomisation code in order to avoid identifying groups. The number will be written on a piece of paper, put in a sealed envelope so that it is not visible, and stored in a double-locked cabinet. A researcher who is not involved in the generation of randomisation codes will open the envelopes sequentially and assign a clinical trial subject to each identification number. The opened envelope will also be stored separately in a double-locked cabinet. The clinical trial subjects will be informed that different medicines are given in phase I and phase II, but will not be told in which phase the therapeutic medicine is given. The process of attaching the randomisation code after manufacturing and packaging of GJD and placebo will be conducted by a researcher who is not involved in assessments to avoid breaking the blind. A researcher will provide medication immediately before each administration time and will check whether or not the subject takes the medication to avoid exchanging medicines between subjects during hospitalisation. To evaluate whether blinding of clinical trial subjects has been properly conducted, an assessment of blinding will be performed using a self-reported questionnaire developed by our research team at the end of the trial, at which point the subjects will be asked in which phase they perceived to have received the medication (as opposed to the placebo).

\section{Sample size}

No previous clinical trials for serum cortisol levels from which sample size could be calculated have been reported; however, we consider a sample size of 24 to be a practical number for detection of clinically important differences between treatment and control groups. This number of subjects (12 per group) is the recommended minimum number per group for pilot studies [23]. We judged that this pilot study recommendation is applicable to feasibility study described here. Based on the results of this study, pilot trials will be needed in order to calculate appropriate sample size.

\section{Statistical analysis}

An independent statistician who will be blinded to the randomised allocation for participants will carry out the statistical analyses. Measured variables including primary and secondary outcomes will be assessed using the full analysis set (FAS) based on intention-to-treat (ITT) principles. The per-protocol (PP) analysis set will be used for the sensitivity analysis. The baseline characteristics for both randomly allocated sequence groups are summarised by means and standard deviations for the continuous variables if satisfying the normal assumption, or median and interquartile range for nonnormal data. Normality will be tested by using the Shapiro-Wilk test. Frequencies and percentages will also be presented for categorical variables. The baseline difference between both sequence groups will be assessed using an independent two-sample $t$ test or a Wilcoxon's rank sum test for continuous variables, and the chi-squared test or Fisher's exact test will be used to verify the randomised allocation for categorical variables.

Changes in serum cortisol levels between the baseline (day 2) and after treatment with GJD or placebo will be calculated to assess the primary outcome according to the following equations:

$$
\begin{aligned}
& C_{G J D}=X_{G J D(E)}-X_{G J D(B L)} \\
& C_{P L}=X_{P L(E)}-X_{P L(B L)}
\end{aligned}
$$

where $C$ is the change in serum cortisol level, $X$ is the serum cortisol level, $E$ is the end point, $B L$ is the baseline, GJD is Gongiin-dan treatment, and $P L$ is placebo.

The primary outcome analysis $\left(\Delta=D_{G J D}-D_{P L}\right)$ will be performed in two stages. First, a linear mixed effect model will be applied to assess the effect of treatment, 
sequence, and period with the random effect for subjects. The carryover effect due to preceding the treatment stage will then be estimated by testing the effect of sequence in the model. The linear mixed model will be modified by confounding the sequence effect to the error term if the carryover effect is not significant. Otherwise, the model will be rebuilt based on data collected in the first phase of the study. An analysis of variance (ANOVA) table for the model will be provided and least square means and their standard errors (95\% confidence intervals) will be reported for each treatment. Second, if the structure of data is balanced and the carryover effect is invalid, simple paired two-sample $t$ tests or Mann Whitney $U$ tests will be performed to evaluate the effect of GJD. The results will be shown as means and standard deviations for each treatment group and mean differences between the two treatments (95\% confidence interval) will be reported.

The analysis of secondary outcomes will be focussed on changes of measures within each treatment group from the baseline to the end of study. Again, the linear mixed effect model will be applied. The level of significance will be set to 0.05 (two-tailed) and all analyses will be performed with PROC MIXED and PROC GLM in SAS software, version 9.4 (SAS Institute Inc., Cary, NC, USA).

\section{Data and safety monitoring}

Regular monitoring will be conducted to ensure quality control of the data according to the planned protocol and standard operating procedures. Monitors blinded to the allocation information will evaluate whether the recruitment procedures are correctly performed and whether the data are adequately recorded according to the protocol in the case report forms. If there are necessary changes to the study methods such as changes to the eligibility criteria, treatment regimens, or follow-up periods, the investigators will discuss these potential changes with independent researchers and statisticians. In the event that severe adverse events and crucial issues occur, the investigators will determine whether these issues are acceptable or if the trial should be amended or ended.

\section{Safety and adverse events}

For the safety of study participants, complete blood counts, liver function tests, and urine analyses will be performed at the screening phase and the end of phases I and II, and vital signs will be checked once daily. Contact information will be provided to all participants for reporting of adverse events at any time. Practitioners will also check for any expected or unexpected adverse events every evening of the hospitalisation periods. Adverse events known to be related to GJD treatment include gastric discomfort, anorexia, nausea, diarrhoea, rash, urticaria due to drug-related allergic reaction, minimal-change disease, and acute focal tubulointerstitial nephritis [24]. Any adverse events reported by participants will be recorded for a causal relationship in the 'Adverse event record table'. A causal relationship between the GJD or placebo treatment and adverse events will be evaluated using a six-grade scale $(1=$ definitely related, $2=$ probably related, $3=$ possibly related, $4=$ probably not related, $5=$ definitely not related, and $6=$ unknown) and the seriousness of any such events will be scored using a three-point scale $(1=$ mild, $2=$ moderate, and $3=$ severe $)$. Any adverse events will be reported in accordance with the regulations of the Institutional Review Board.

\section{Ethics}

This study complies with the principles of the Declaration of Helsinki and Good Clinical Practice guidelines. The protocol has been reviewed and approved by the Institutional Review Board of the Daejeon Korean Medicine Hospital of Daejeon University (DJOMC-127-1) and is registered with the national clinical trial registry Clinical Research Information Service, which is a primary registry of the World Health Organisation International Clinical Trials Registry Platform (http://apps.who.int/trialsearch/ Default.aspx; CRIS registration No. KCT0001681). Any protocol modifications will be approved by the Institutional Review Board of the Daejeon Korean Medicine Hospital of Daejeon University.

Written informed consent will be obtained from all study participants prior to enrolment into the study and participants will be given the option to decline to participate or withdraw at any time without disadvantage.

\section{Discussion}

Deciding on the appropriate primary outcome of a trial is one of the challenges of trial design. The primary outcome in this study protocol is serum cortisol level measured at $30 \mathrm{~min}$ after waking. Cortisol is closely related to fatigue and blunted cortisol levels are primarily observed in patients with fatigue [25-27]. Although fatigue is a subjective symptom, cortisol levels were selected as the primary variable over the questionnaire-derived results.

Cortisol should be measured at the same time every day due to circadian rhythm. Cortisol levels may, moreover, be affected by dietary supplements [28], caffeine [29], and intensive exercise [30], as well as foods with a high sugar, fat, or protein content $[12,13]$. The crossover method and participant hospitalisation were implemented in this study to minimise confounding factors and other possible bias [31].

To determine perceived symptoms and to compare these with objective outcomes, the results of self-reported questionnaires including the FSS-K, BFI-K, KMLSEQ, and 
daily fatigue and sleep evaluation questionnaires will be evaluated.

Epinephrine, norepinephrine, and HRV are related to the autonomic nervous system and as such are stimulated as a response to stress in the human body. Most individuals experiencing fatigue have hyperactive sympathetic nerves and hypoactive parasympathetic nerves $[32,33]$, and variations in autonomic nerve activity in the fatigue state can be observed as changes in serum concentrations of epinephrine and norepinephrine as well as in HRV [34].

In comparisons of normal individuals to those experiencing fatigue, it has been reported that circulating oxidative stress increases with fatigue, but that antioxidant levels decrease [35-37]. Immunity disturbances have been researched for potential involvement in chronic fatigue syndrome and analysis of immunological factors are, therefore, also included in this study [38, 39].

This is a feasibility study and is, thus, not powered to determine treatment effectiveness. As appropriate sample size could not be determined due to a lack of similar previous studies, the minimal sample size considered clinically significant was defined. The results of this study may, therefore, affect generalisability in other environments. Moreover, because fatigue will be artificially induced in healthy people in this study, the findings would not represent chronic fatigue, which is more clinically relevant. Further study with exact sample sizes in patients complaining of chronic fatigue would be necessary and could be designed based on the results from this study. This study protocol represents a unique opportunity to enhance our understanding of fatigue and the effects GJD on fatigue in terms of endocrine and immunological mechanisms and may, therefore, serve to improve future treatment strategies for chronic fatigue.

\section{Trial status}

Recruitment for this trial opened in September 2015 and will close in December 2015. At the time of manuscript submission, the trial was in the recruitment phase.

\section{Acknowledgements}

This study is supported by the Korea Institute of Oriental Medicine (K16121).

\section{Authors' contributions}

CGS conceived and designed the study protocol. MJS drafted the manuscript and YEK helped to draft the manuscript, while HJL and BK made substantial contributions to designing the study protocol. CGS and JHL had the final responsibility for the decision to submit for publication. All the authors have read and approved the final manuscript for submission.

\section{Competing interests}

The authors declare that they have no competing interests.

\section{Author details}

${ }^{1}$ Clinical Research Division, Korea Institute of Oriental Medicine, 1672 Yuseongdae-ro, Yuseong-gu, Daejeon 34054, Republic of Korea. ${ }^{2}$ Liver and Immunology Research Center, Daejeon Korean Medicine Hospital of Daejeon
University, 176-9, Daeheung-ro, Jung-gu, Daejeon 34929, Republic of Korea. ${ }^{3}$ Mibyeong Research Center, Korea Institute of Oriental Medicine, 1672 Yuseongdae-ro, Yuseong-gu, Daejeon 34054, Republic of Korea. ${ }^{4} \mathrm{KM}$ Fundamental Research Division, Korea Institute of Oriental Medicine, 1672 Yuseongdae-ro, Yuseong-gu, Daejeon 34054, Republic of Korea. ${ }^{5}$ Korean Medicine Life Science, University of Science \& Technology, Campus of Korea Institute of Oriental Medicine, 1672 Yuseongdae-ro, Yuseong-gu, Daejeon, Republic of Korea.

Received: 2 December 2015 Accepted: 6 August 2016

Published online: 22 August 2016

\section{References}

1. Sharpe M, Archard L, Banatvala J, Borysiewicz L, Clare A, David A, et al. A report—chronic fatigue syndrome guidelines for research. J R Soc Med. 1991;84:118-21.

2. Son C. Review of the prevalence of chronic fatigue worldwide. J Korean Orient Med. 2012;33:25-33.

3. Reynolds K, Vernon S, Bouchery E, Reeves W. The economic impact of chronic fatigue syndrome. Cost Eff Resour Alloc. 2004;2:1-9. doi:10.1186/1478-7547-2-4.

4. Jorgensen R. Chronic fatigue: an evolutionary concept analysis. J Adv Nurs. 2008;63:199-207. doi:10.1111/j.1365-2648.2008.04649.x.

5. Adams D, Wu T, Yang X, Tai S, Vohra S. Traditional Chinese medicinal herbs for the treatment of idiopathic chronic fatigue and chronic fatigue syndrome. Cochrane Database Syst Rev. 2009;4:CD006348.

6. Xu Y, Chen Y, Li P, Wang XS. Ren Shen Yangrong Tang for fatigue in cancer survivors: a phase I/II open-label study. J Altern Complement Med (New York, NY). 2015;21:281-7. doi:10.1089/acm.2014.0211.

7. Wang Y, Li X, Liu J, Luo H, Ma L, Alraek T. Traditional Chinese medicine for chronic fatigue syndrome: a systematic review of randomized clinical trials. Complement Ther Med. 2014;22:826-33. doi:10.1016/j.ctim.2014.06.004.

8. Chen R, Moriya J, Yamakawa J, Takahashi T, Kanda T. Traditional Chinese medicine for chronic fatigue syndrome. Evid Based Complement Alternat Med. 2010;7:3-10. doi:10.1093/ecam/nen017.

9. Hong S, Lee J, Lee J, Lee H, Kim H, Lee S, et al. The traditional drug Gongjin-Dan ameliorates chronic fatigue in a forced-stress mouse exercise model. J Ethnopharmacol. 2015;168:268-78. doi:10.1016/j.jep.2015.04.001.

10. Chen J, Hsiang $C$, Lin $Y$, Ho T. Deer antler extract improves fatigue effect through altering the expression of genes related to muscle strength in skeletal muscle of mice. Evid Based Complement Alternat Med. 2014;2014: 540580. doi:10.1155/2014/540580

11. Li S, Chen Z, Zhang C. Effect of tao-hong-si-wu-tang, a traditional Chinese herbal medicine formula, on physical fatigue in mice. Afr J Tradit Complement Altern Med. 2012;10:60-5.

12. MacKenzie T, Comi R, Sluss P, Keisari R, Manwar S, Kim J, et al. Metabolic and hormonal effects of caffeine: randomized, double-blind, placebocontrolled crossover trial. Metabolism. 2007;56:1694-8. doi:10.1016/j.metabol. 2007.07.013

13. Gibson E, Checkley S, Papadopoulos A, Poon L, Daley S, Wardle J. Increased salivary cortisol reliably induced by a protein-rich midday meal. Psychosom Med. 1999;61:214-24.

14. Krupp L, LaRocca N, Muir-Nash J, Steinberg A. The fatigue severity scale. Application to patients with multiple sclerosis and systemic lupus erythematosus. Arch Neurol. 1989;46:1121-3.

15. Mendoza T, Wang X, Cleeland C, Morrissey M, Johnson BA, Wendt JK, et al. The rapid assessment of fatigue severity in cancer patients: use of the Brief Fatigue Inventory. Cancer. 1999;85:1186-96.

16. Yun Y, Wang X, Lee J, Roh J, Lee C, Lee W, et al. Validation study of the Korean version of the Brief Fatigue Inventory. J Pain Symptom Manage. 2005;29:165-72. doi:10.1016/j.jpainsymman.2004.04.013.

17. Kim I, Choi H, Kim B. Psychometric properties of Korean Version of Modified Leeds Sleep Evaluation Questionnaire (KMLSEQ). Korean J Rehabil Nurs. 2014;17:10-7.

18. Hayashi I, Morishita Y, Imai K, Nakamura M, Nakachi K, Hayashi T. Highthroughput spectrophotometric assay of reactive oxygen species in serum. Mutat Res. 2007;631:55-61. doi:10.1016/j.mrgentox.2007.04.006.

19. Green L, Wagner D, Glogowski J, Skipper P, Wishnok J, Tannenbaum S. Analysis of nitrate, nitrite, and [15 N]nitrate in biological fluids. Anal Biochem. 1982;126:131-8. 
20. Kamal A, Gomaa A, el Khafif M, Hammad A. Plasma lipid peroxides among workers exposed to silica or asbestos dusts. Environ Res. 1989;49:173-80.

21. Kambayashi Y, Binh N, Asakura H, Hibino Y, Hitomi Y, Nakamura H, et al. Efficient assay for total antioxidant capacity in human plasma using a 96-well microplate. J Clin Biochem Nutr. 2009;44:46-51. doi:10.3164/jcbn.08-162.

22. Wheeler C, Salzman J, Elsayed N, Omaye S, Korte Jr DW. Automated assays for superoxide dismutase, catalase, glutathione peroxidase, and glutathione reductase activity. Anal Biochem. 1990;184:193-9.

23. Julious S. Sample size of 12 per group rule of thumb for a pilot study. Pharm Stat. 2005;4:287-91. doi:10.1002/pst.185.

24. Lee $\mathrm{K}$, Jeong $\mathrm{H}$, Rhee $\mathrm{H}$. A patient with minimal change disease and acute focal tubulointerstitial nephritis due to traditional medicine: a case report and small literature review. Explore (NY). 2014;10:319-23. doi:10.1016/j. explore.2014.06.001.

25. Crofford L, Young E, Engleberg N, Korszun A, Brucksch C, McClure L, et al. Basal circadian and pulsatile ACTH and cortisol secretion in patients with fibromyalgia and/or chronic fatigue syndrome. Brain Behav Immun. 2004;18: 314-25. doi:10.1016/j.bbi.2003.12.011.

26. Roberts A, Wessely S, Chalder T, Papadopoulos A, Cleare AJ. Salivary cortisol response to awakening in chronic fatigue syndrome. Br J Psychiatry. 2004; 184:136-41.

27. Demitrack M, Dale J, Straus S, Laue L, Listwak SJ, Kruesi MJ, et al. Evidence for impaired activation of the hypothalamic-pituitary-adrenal axis in patients with chronic fatigue syndrome. J Clin Endocrinol Metab. 1991;73:1224-34.

28. Bhathena S, Berlin E, Judd J, Kim Y, Law J, Bhagavan H, et al. Effects of omega 3 fatty acids and vitamin $\mathrm{E}$ on hormones involved in carbohydrate and lipid metabolism in men. Am J Clin Nutr. 1991;54:684-8.

29. Lovallo W, Farag N, Vincent A, Thomas T, Wilson M. Cortisol responses to mental stress, exercise, and meals following caffeine intake in men and women. Pharmacol Biochem Behav. 2006;83:441-7. doi:10.1016/j.pbb.2006.03.005.

30. Robson P, Blannin A, Walsh N, Castell L, Gleeson M. Effects of exercise intensity, duration and recovery on in vitro neutrophil function in male athletes. Int J Sports Med. 1999:20:128-35. doi:10.1055/s-2007-971106.

31. Powell D, Liossi C, Moss-Morris R, Schlotz W. Unstimulated cortisol secretory activity in everyday life and its relationship with fatigue and chronic fatigue syndrome: a systematic review and subset metaanalysis. Psychoneuroendocrinology. 2013;38:2405-22. doi:10.1016/j. psyneuen.2013.07.004

32. Fagundes C, Murray D, Hwang B, Gouin J, Thayer J, Sollers J, et al. Sympathetic and parasympathetic activity in cancer-related fatigue: more evidence for a physiological substrate in cancer survivors. Psychoneuroendocrinology. 2011; 36:1137-47. doi:10.1016/j.psyneuen.2011.02.005.

33. Tak L, Riese H, de Bock G, Manoharan A, Kok I, Rosmalen J. As good as it gets? A meta-analysis and systematic review of methodological quality of heart rate variability studies in functional somatic disorders. Biol Psychol. 2009:82:101-10. doi:10.1016/j.biopsycho.2009.05.002.

34. Strüder H, Hollmann W, Platen P, Donike M, Gotzmann A, Weber K. Influence of paroxetine, branched-chain amino acids and tyrosine on neuroendocrine system responses and fatigue in humans. Horm Metab Res. 1998;30:188-94.

35. Kennedy G, Spence V, McLaren M, Hill A, Underwood C, Belch JJ. Oxidative stress levels are raised in chronic fatigue syndrome and are associated with clinical symptoms. Free Radic Biol Med. 2005;39:584-9. doi:10.1016/j. freeradbiomed.2005.04.020.

36. Vecchiet J, Cipollone F, Falasca K, Mezzetti A, Pizzigallo E, Bucciarelli T, et al. Relationship between musculoskeletal symptoms and blood markers of oxidative stress in patients with chronic fatigue syndrome. Neurosci Lett. 2003;335:151-4.

37. Kim J, Park E, Min H, Kang M-H. Relationships of plasma homocysteine concentration and oxidative stress markers in Korean college students. Korean J Nutr. 2010:43:443-52.

38. Pariante C. Chronic fatigue syndrome and the immune system: 'findings in search of meanings'. Brain Behav Immun. 2009;23:325-6. doi:10.1016/j.bbi. 2009.01.006.

39. Lorusso L, Mikhaylova S, Capelli E, Ferrari D, Ngonga GK, Ricevuti G. Immunological aspects of chronic fatigue syndrome. Autoimmun Rev. 2009; 8:287-91. http://dx.doi.org/10.1016/j.autrev.2008.08.003.

40. Ministry of Food and Drug Safety.The Korean Pharmacopoeia. 11th ed. http://drug.mfds.go.kr/html/menuLinkBody.jsp?p_menuld=0514 Accssed 18 Aug 2016.

\section{Submit your next manuscript to BioMed Central and we will help you at every step:}

- We accept pre-submission inquiries

- Our selector tool helps you to find the most relevant journal

- We provide round the clock customer support

- Convenient online submission

- Thorough peer review

- Inclusion in PubMed and all major indexing services

- Maximum visibility for your research

Submit your manuscript at www.biomedcentral.com/submit
Biomed Central 